\title{
Mechanical Behavior and Fracture Toughness Evaluation of Multiphase Polymer Nanocomposites Using Impact and $J$-Integral via Locus Method
}

\author{
Bishnu P. Panda, Smita Mohanty, and S. K. Nayak \\ Laboratory for Advanced Research in Polymeric Materials (LARPM), Central Institute of Plastics Engineering \\ and Technology, Bhubaneswar, Orissa 751024, India
}

Correspondence should be addressed to S. K. Nayak; papers.journal@gmail.com

Received 12 August 2013; Accepted 8 October 2013

Academic Editors: M. Brünig, R. Hong, Y.-C. Ke, P.-y. Manach, and B. Sun

Copyright (c) 2013 Bishnu P. Panda et al. This is an open access article distributed under the Creative Commons Attribution License, which permits unrestricted use, distribution, and reproduction in any medium, provided the original work is properly cited.

\begin{abstract}
Fracture behaviors of fibrillar silicate clay (MMT) filled thermoplastic polyolefin (TPO) containing polypropylene (PP) blended with ethylene-propylene-diene monomer (EPDM) were systematically investigated using impact test method and $J$-integral by locus method. Drastic increase in impact strength is observed for all developed compositions and generally shows higher value for the selected phases containing dispersed nanoclay in PP matrix. A fracture mechanics approach has been adopted by mode I test, and the effects of specimen geometry have been investigated. Increase in interlaminar fracture energy value, $G_{c}$, and $J$ integral value, $J_{c}$, is marked as the crack propagated through the composite; that is, a rising " $R$-curve" is observed. Toughness measurements revealed that the fracture toughness increased with increasing clay content reaching maximum at $3 \mathrm{wt} \%$ of clay than pure PP. Moreover, enhancement of fracture toughness was more remarkable than that of stiffness. The fracture surfaces taken from different specimens were observed for exploring the fracture mechanisms using transmission electron microscopy (TEM) revealed a strong particle-matrix adhesion.
\end{abstract}

\section{Introduction}

Polypropylene (PP) offers a very attractive combination of physical and mechanical properties at a relatively low cost, which makes it a versatile material with continuously increasing applications. However, not all the characteristics of this material are suitable for common service conditions. For instance, $\mathrm{PP}$ exhibits poor low-temperature impact resistance because of its low temperature $\left(T_{q}\right)$ and high crystallinity. In order to overcome these limitations, elastomers such as ethylene-propylene random copolymer (EPR) [1], ethylenepropylene-diene terpolymer (EPDM) [2], and poly(ethyleneco-1-octene) [3] among others $[4,5]$ have been added to $\mathrm{PP}$, as impact modifiers. Further, due to incompatibility of PP with these elastomers, functionalized polymers have been used as blend compatibilizers to improve interfacial adhesion. Layered silicate clays are also used as fillers in polymers which have gained considerable attention due to the ability to achieve exceptional property enhancements at very low level loading. Layered silicate clays such as montmorillonite (MMT) have attracted much attention because of their unique properties, such as large aspect ratio, high surface area, and low cost. Addition of low level loading of these silicate clays to polymers markedly improves their tensile strength, stiffness, and heat resistance and facilitates processing thus reducing component weight. However, in order to attain a qualitatively fine dispersion of the nanofiller within the matrix, a surface treatment of the filler should be considered or a polymeric compatibilizer should be added during melt mixing.

In order to minimize the number of experiments, it is useful to evaluate toughness at least under two extreme conditions, that is, under impact conditions and fracture toughness by SENT test methods. Toughness of polymers is often measured using Charpy and Izod impact test. A method for the determination of fracture toughness has been developed by Marshall et al. [6] by calculating strain energy release rate $\left(G_{c}\right)$ directly from the results of impact tests on 
multiple specimens with different notch depths. Plati and William [7] reported that critical strain energy release rate $G_{c}$ can be easily deduced from the absorbed energy measurement with the assumption of elastic deformation from impact test. Sanporean et al. [8] reported significant improvement in fracture stress and fracture energy by incorporation of $3 \mathrm{wt} \%$ $\mathrm{TiO}_{2}$ clay in PP/EPDM(70/30)blend. Result of impact test involves measurement of energy $(U)$ to break notched specimen generally divided by ligament area $(A)$ to provide surface energy $(U / A)$. In ductile polymers such as rubber-toughened plastics, there is a considerable amount of energy dissipation ahead of the crack tip due to plastic deformation during crack propagation. Hence, the $J$-integral concept was introduced by Rice to predict the fracture behavior of ductile polymers [9]. The $J$-integral is an energy input which can be used as a design parameter. Many different methodologies are used to determine $J_{c}$-integral at crack initiation for ductile polymers. One successful method is the locus method developed by Kim and Joe $[10,11]$. The technique is based on the locus of crack initiation points on the load-displacement curve by visual observation. This method has been successfully utilized for highly deformable materials like Santoprene [10], Polystyrene and PP homopolymer [11], NR/SBR/BR blend system [12] and thermoplastic copoly (ether ester) elastomer, Acrylonitrile butadiene styrene blends [13].

In this study, we seek to ascertain the mechanical behavior and deformation mechanisms associated with the increase in toughness and stiffness as a result of the inclusion of organically modified nanoparticles within an elastomeric modified PP matrix. Consideration was also made of the effect of these deformation processes upon strength. To promote exfoliation of clay platelets, polypropylene-graftedmaleic anhydride (PP-g-MA) material has been premixed with the organoclay prior to melt processing. The effect of nanoclay on mode I type fracture toughness has been studied and the characteristics of failure are examined by studying the structural changes taking place within the nanocomposites employing TEM techniques. Furthermore, the fracture toughness behavior of these nanocomposites is compared.

\section{Methodology}

2.1. Fracture Toughness by Impact Test Method. The total energy $(U)$ absorbed by the specimen related to fracture energy of the material expressed in terms of "critical strain energy release rate" $G_{c}$ for situation like size of yield zone ahead of the crack tip is expressed by the following equation:

$$
G_{c}=\frac{U}{B D \emptyset},
$$

where $B$ is specimen thickness; $D$ is specimen width; $\emptyset=$ $c \div d c / d(a / w)$ is a calibration factor which depends on the length of the notch and the size of the sample; $c$ is specimen compliance; $a$ is notch length; $w$ is specimen width; $G_{c}$ value obtained from the slope of plot made by $U$ versus $B D \emptyset$ by series of the specimens with different notch lengths.
2.2. Determination of Fracture Energy by Locus Line Method. Having varying crack sizes, the fracture energy has been evaluated in terms of the critical $J$-integral value, $J_{c}$, utilizing the crack initiation locus line method [10,11]. The method determines $J_{c}$ as follows:

$$
J_{c}=-\frac{1}{B} \frac{\Delta U_{c}}{\Delta a},
$$

where $U_{c}$ is the enclosed area between the loading line and the locus line. Here we have used specimens (width and thickness 12.7 and $3.2 \mathrm{~mm}$, resp.) having varying crack sizes to evaluate the fracture energy in terms of the critical $J$-integral value, $J_{c}$, utilizing the crack initiation locus line method. In preparing the test specimens, the initial crack was made by slotting and a sharp crack was then introduced by pushing a razor blade into the blunt notch. The $a / w$ ratio varied from 0.2 to 0.3. Load-displacement graphs were recorded and crack initiation points were marked on each loading line during the test. The crack tip area was illuminated from behind the bend fixture so that the crack initiation points could be clearly seen. Similar to the crack initiation point, maximum load point in the load versus deflection curves can be used to measure fracture toughness for calculation of $R$-curves. Here $U_{c}$ is replaced with $U_{R}$ which is the area enclosed by the locus line of maximum points of the load-displacement curves and the $x$-axis.

\section{Experimental}

3.1. Materials. Polypropylene copolymer Repol B120MA of density 0.96 and Melt Flow Index $12 \mathrm{~g} / 10 \mathrm{~min}\left(230^{\circ} \mathrm{C} / 2.16 \mathrm{~kg}\right.$, ASTM D 1238) was supplied by Reliance Petrochemicals. Ethylene-propylene-diene rubber (EPDM) in pellets, medium molecular weight with ethylidene norbornene as termonomer (4.5\%), ethylene content of about $68 \%$, and medium cure rate, was supplied by Dupont Dow elastomers under the trade name Nordel IP 4770. Sodium montmorillonite (Na-MMT) with cationic exchange capacity (CEC) of 92 mequiv/100 g was purchased from Southern Clay Products Inc., USA. Aminopropyltriethoxysilane $\mathrm{N}$-(2-aminoethyl)3 -aminopropyltrimethoxy silane (98\%, Merck) and triethylamine $\left(\mathrm{Et}_{3} \mathrm{~N}, 99 \%\right.$, Merck) were used as coupling agent and surface grafting catalyst, respectively. Methoxy propyl acetate (MPA, 96\%), butyl acetate (99\%), and toluene (98\%) were obtained from Aldrich chemicals.

3.2. Surface Modification of Na-MMT Nanoparticle. 12 grams of Na-MMT nanoparticles were kept in a vacuum chamber for $24 \mathrm{~h}$ at $100^{\circ} \mathrm{C}$ and then dispersed in $500 \mathrm{~mL}$ anhydrous toluene with the aid of ultrasonication for $30 \mathrm{~min}$. Triethyl Amine of quantity $1.5 \mathrm{~mL}$ used as a catalyst and $15 \mathrm{wt} \%$ $(1.5 \mathrm{~mL})$ aminopropyltrimethoxysilane (APS) were added, respectively, with constant stirring at $300 \mathrm{rpm}$ for 1 hour. The mixture was then refluxed for $5 \mathrm{~h}$ under nitrogen atmosphere. Modified particles were filtered under suction and physically adsorbed. APS compound on the modified surface of nanoparticles was washed with ethanol for 4-5 times and then dried in a low pressure oven at $80^{\circ} \mathrm{C}$ for $12 \mathrm{hrs}$. 
3.3. Preparation of Blend and Its Nanocomposites. Different blends and nanocomposites were formed containing melt compounding mixture of PP and EPDM, and a masterbatch material containing maleated PP (MA-g-PP, contents 2$5 \mathrm{phr}$ ), and an organically modified MMT clay (1-5 wt \%). Compounding was carried out using microcompounder (Xplore, DSM, Netherlands) at screw speed of 60 RPM and maintained constant torque level of $80 \mathrm{Nm}$ in all cases. The resulting temperature during the process of material was maintained at $190^{\circ} \mathrm{C}$ followed by annealing in a vacuum oven at $80^{\circ} \mathrm{C}$ for a 4 -hour duration. The first series of $\mathrm{PP}$ with EPDM blend was obtained at level varying from 10 to $50 \mathrm{wt} \%$ in PP matrix. Extruded nanocomposite pellets were dried and then formed into standard tensile specimens (ASTM D638, Type I) and impact specimens by Izod test (ASTM D256) bars in an Xplore injection molding machine using barrel temperature $190^{\circ} \mathrm{C}$, injection pressure of $40 \mathrm{bar}$, and holding pressure of $30 \mathrm{bar}$.

3.4. Mechanical Properties. An instrumented Izod type impact testing apparatus, CEAST, Italy, was used to study the impact strength of all samples and total energy observed by measuring potential energy lost as per ASTMD 256 standard. The hammer had a $3.65 \mathrm{~kg}$ mass and the impact velocity was $1.92 \mathrm{~m} / \mathrm{s}$. Impact specimens of size $76 \times 12.7 \times 3.2 \mathrm{~mm}$ were machined and they were subsequently notched using a notch cutter to produce notch lengths of $1.5,2.5,3.5$, and $4.5 \mathrm{~mm}$, respectively. All specimens precracked by saw cut and then were repeatedly slide by razor blade to provide sufficient sharp micronotch under normal load using custom made rig. Tensile properties were measured as per ASTM-D 638 with gauge length of $50 \mathrm{~mm}$ at a crosshead speed of $50 \mathrm{~mm} / \mathrm{min}$ by using universal testing machine (3382 Instron, UK). Tests for determination of mechanical properties were carried out in a standard temperature of $23^{\circ} \mathrm{C} \pm 2^{\circ} \mathrm{C}$ and $50 \% \pm 2 \% \mathrm{RH}$.

3.5. Single Edge Notch Bending Method (SENB). J-integral was calculated using SENB test method with nominal dimension, $D=12.7 \mathrm{~mm}, B=3.2 \mathrm{~mm}$, and $L=76 \mathrm{~mm}$ and applied conventional linear elastic fracture mechanics method. Test has been conducted as per ASTM E399-90 by universal testing machine (Instron 3382) at a speed of $1 \mathrm{~mm} / \mathrm{min}$. The initial crack length was varied $1.5,2.5,3.5$, and $4.5 \mathrm{~mm}$ for different specimen and load-displacement graphs were recorded.

3.6. Transmission Electron Microscopy (TEM). TEM specimens were cut from the impact fractured plaque with a Reichert Ultracut microtome. The films were placed onto the $\mathrm{Cu}$ grids and then placed into the specimen chamber of a transmission electron microscope (Jeol JEM1400).

The morphology of nanocomposite was investigated using a Philips CM300 transmission microscope (TEM:FEI Company, USA) at an accelerating voltage of $200 \mathrm{KV}$. Thin sections with thickness of $80 \mathrm{~nm}$ were cut from the fractured specimen by using a Leica EM UC7 ultramicrotome (equipped with a diamond knife under cryogenic condition) for subsequent TEM observations.

\section{Result and Discussion}

4.1. Impact Properties. Figure 1 depicts influence of $\mathrm{Cl}-30 \mathrm{~B}$ clay and elastomer content on notched Izod impact strength of PP matrix. The presence of $40 \%$ elastomer reasonably increased impact strength up to $175 \%$ as compared with neat PP. This resulted in phenomenal transition from brittle to ductile behavior of PP matrix due to the presence of small elastomeric domains leading to more toughness [14]. Further, at $50 \mathrm{wt} \%$ of EPDM, there was no break in the sample. The sample with $40 \mathrm{wt} \%$ of EPDM was taken for preparation of nanocomposite. However, there was a decline in impact strength beyond $40 \%$ of EPDM content. This may be due to an increase in rubber particle size which contributed inhomogenous miscibility in the PP matrix resulting in a decreased impact value. In case of the nanocomposite containing up to $40 \mathrm{wt} \%$ of elastomer, the addition of clay leads to an increase in the Izod impact values reaching a maximum at $3 \mathrm{wt} \%$ as shown in Figure 1(a). This may be due to the ability of filler to support stress transferred from the filler to matrix. Decrease in impact strength is usually expected with increasing filler content; however, opposite trend was observed for the nanocomposite which can be explained possibly in terms of morphological changes induced by the dispersion of clay particles. The increase in impact strength may be ascribed due to reduction in the size of elastomer particle due to the barrier effect of clay. At $5 \mathrm{wt} \%$ clay, there is a little decline in the impact strength observed which is probably due to agglomeration of nanoparticles.

Further, at fixed elastomer loading of $40 \mathrm{wt} \%, \mathrm{PP} / \mathrm{EPDM}$ blend nanocomposite incorporation of MAPP results in higher impact strength than the blends without MAPP (Figure 1(b)). This can be attributed to improved dispersion of nanoclay at the interface of PP/EPDM matrix. MAPP not only improves the miscibility between organically modified clay and PP but also aids in expanding the clay galleries and enabling insertion of polymer chains leading to formation of intercalated nanocomposites structure. The optimum impact property with addition of MAPP is probably due to the fact that modified clay is polar in nature with two hydroxyl groups in its structure which interacts more easily with carboxyl group of MAPP while the PP part of the compatibilizer becomes compatible with the PP macromolecular chains [15]. Impact strength of blend matrix increased up to $660 \mathrm{~J} / \mathrm{m}$ which indicates toughening effect of nanoclay layers within the blend matrix. Incorporation of MAPP further reduces the interfacial energy of both phases and provides a reactive site for intercalation of clay. The differences in chemical structure and organic modifier used in the nanoclay played a vital role in dispersion of layered silicates within the blend matrix.

4.2. Tensile Behavior. Figure 2(a) shows the effect of clay and EPDM content on the modulus of PP/EPDM blend nanocomposites. It is evident that the addition of clay to PP containing MAPP as compatibilizer results in a substantial increase in stiffness at low clay concentrations, that is, 1$3 \mathrm{wt} \%$, and with the increase at higher clay concentrations, the effect is less pronounced. Similar trends are also observed for PP/EPDM blend nanocomposites [16, 17]. Effects of 


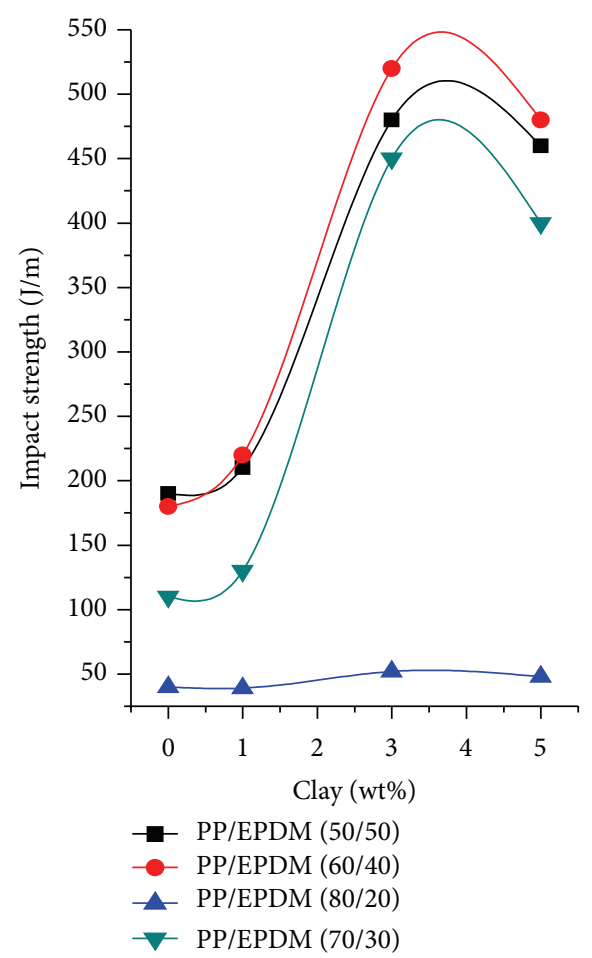

(a)

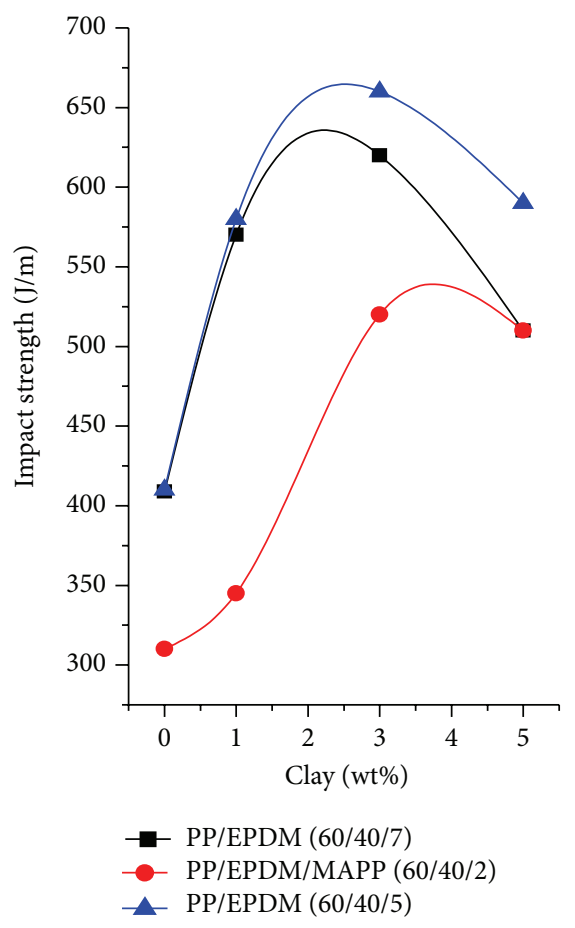

(b)

FIGURE 1: Effect of clay and elastomer content on notched impact strength of PP/EPDM/blend nanocomposites: (a) nanocomposites containing variable content of elastomer and (b) nanocomposites containing variable content of MAPP compatibilizer.

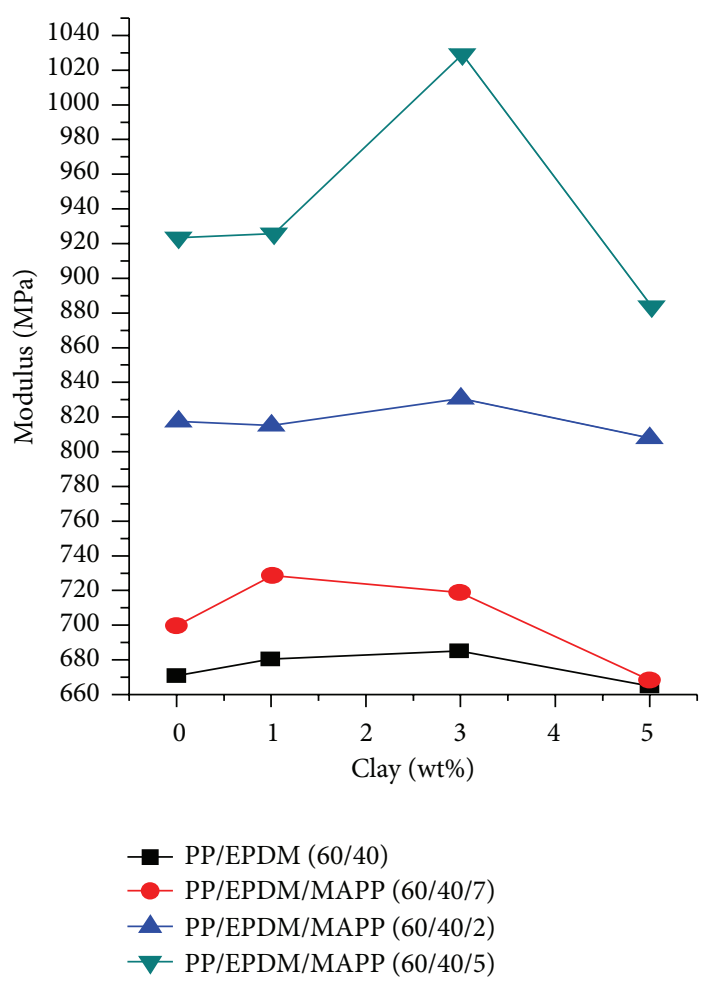

(a)

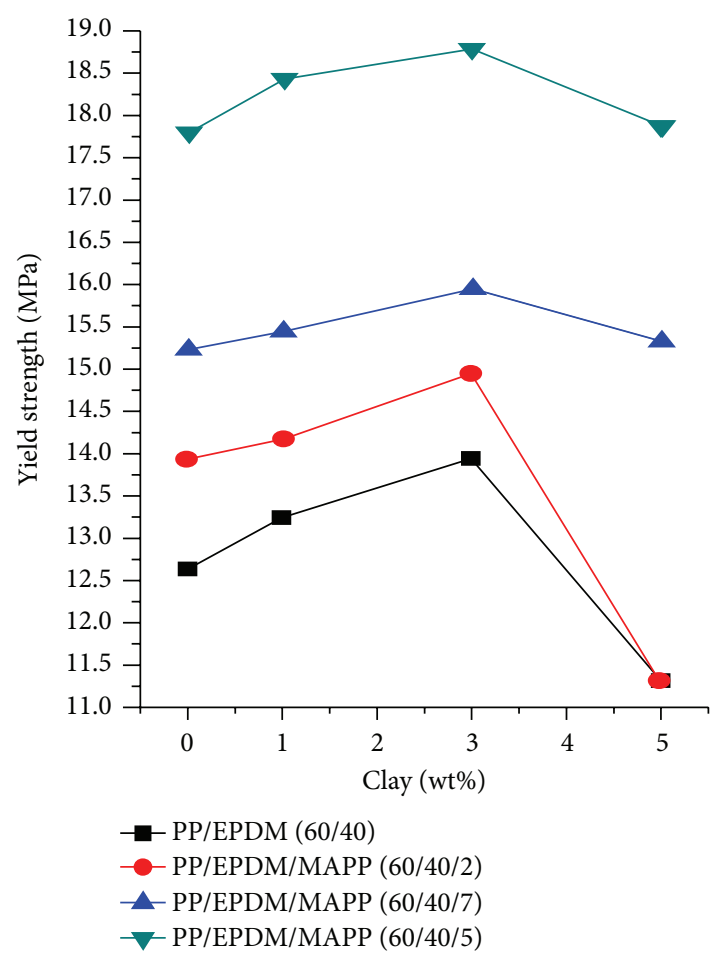

(b)

FIGURE 2: Effect of clay and elastomer content on (a) the tensile modulus and (b) yield strength of PP/EPDM nanocomposites. 


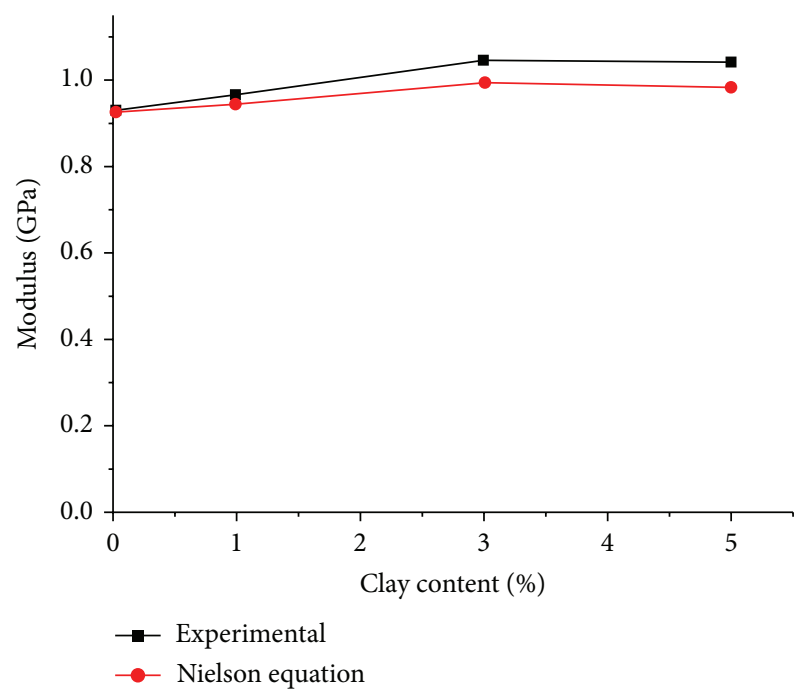

FIGURE 3: Variation of Young's modulus with clay content.

other factors such as reduced platelet orientation and/or the increased amount of PP-g-MA are needed to achieve the better dispersion of silicate platelets in the PP matrix and, as a consequence, improved stiffness of PP nanocomposite. At $5 \mathrm{phr}$ of MAPP, it is observed that an increase of $56 \%$ of tensile modulus was observed in compatibilized PP/EPDM blend nanocomposite with the loading of $3 \mathrm{wt} \%$ clay as compared with the blend matrix of $60: 40$ blend ratios. This behavior is probably due to the fact that PP is inherently stiff and incorporation of compatibilizer with presence of elastomer and clay results in an increase in the crystallinity of the virgin polymer with a consequent increase in tensile modulus $[8,18]$. Addition of MAPP in the blend also reduces the interfacial energy of both phases, thereby increasing the compatibility. It is interesting to note that $5 \mathrm{phr}$ of graft polymer is sufficient for interfacial saturation $[19,20]$. The equilibrium concentration of MAPP at which the domain size levels are critical is called critical micelle concentration(CMC), that is, the concentration above which micelles are formed. The interfacial tension is directly proportional to domain size $[21,22]$. Conversely, a decrease in tensile strength of the blend was also noticed irrespective of addition of MAPP. This reduction in tensile strength reveals inherently weak strength of PP, which has been widely reported by various authors for blends of polyesters with semicrystalline polyolefins [23, 24].

Figure 2(b) shows the influence of clay and elastomer content on the yield strength for PP/EPDM/MAPP blend nanocomposites with different wt $\%$ of clay. Unlike the modulus, adding clay to PP/EPDM blends results in a slight increase in yield strength with addition of clay and, as expected, adding elastomer in the absence of compatibilizer results decrease in the yield strength of the nanocomposites. Addition of clay beyond $3 \mathrm{wt} \%$ does not significantly change the yield strength of these nanocomposites. Incorporation of MAPP and clay further reduces the interfacial energy of both phases and provides a reactive site for intercalation of clay to the matrix. In the optimized compatibilized blend, addition of the above clay concentration reduced the yield strength. This enhancement of tensile modulus and tensile strength is due to the reduction in molecular chain mobility. The differences in chemical structure and organic modifier used in the nanoclay played a vital role in the dispersion of layered silicates within the blend matrix.

Figure 3 shows measured values of Young's modulus of nanocomposites at different $\mathrm{wt} \%$ of clay studied compared to predictions of the Nielsen's modified Kerner's equation. For composites, Young's modulus is evaluated by using the following expression [25]:

$$
E_{c}=E_{m}\left(\frac{1+A B \emptyset_{p}}{1-B \psi \emptyset_{p}}\right)
$$

where $A$ is a constant dependent on the geometry of the filler phase and the Poisson's ratio of the matrix, $B$ is a constant dependent on the relative stiffness of the particulate filler and the matrix, and $\emptyset_{P}$ is dependent on the packing fraction of the filler. Taking Poisson's ratio of the matrix to be 0.4 [25], $A$ is calculated to be 1.25 and $B$ is calculated to be close to 1 due to the high filler to matrix modulus ratio. Taking a packing fraction of 0.37 [26] for random close packing of the agglomerates, the value of the predicted modulus can be seen as the dotted line in Figure 3. Nielsen's modified Kerner's equation considers individual particulate effect rather than the iso-stress conditions more appropriate to the laminates. Furthermore, it takes into account the particle size distribution through the maximum packing fractions. When a packing fraction value of 0.37 for random close packing with agglomerates was adopted, the predicted Young's modulus data correlated well with the experimental data.

\subsection{Toughness Measurement}

4.3.1. Fracture Toughness in Impact Test Method. Figure 4(a) shows typical plot of total energy $U$ versus ligament area $(B D \emptyset)$ for each initial crack size of the PP/EPDM blend as well as PP/EPDM/Cl 30B blend nanocomposite. The result implies linear relationship between $G_{c}$ for both binary blends and ternary composites. It is shown that addition of clay to the PP/EPDM/MAPP blends exhibited higher $G_{c}$ values than that of PP and PP/EPDM blends, which implies incorporation of clay enhanced toughness of the binary blends [27]. Enhancement of toughness was more prominently observed at $3 \mathrm{wt} \%$ of clay loading. This may be the result of better enhancement of compatibilization and adhesion effect of nanoclay due to the presence of the MAPP in the PP/EPDM matrix [28]. Presence of the compatibilizer and elastomer in the nanocomposite undergoes cavitation, which releases triaxiality at the notch and permits deformation by shear yielding. It is worth noting that addition of clay as filler is expected to make high embrittlement to PP matrix. This implied synergistic toughening effect by addition of clay into the PP/EPDM matrix with formation of encapsulation may be due to better interfacial interaction and unique molecular microstrength. 


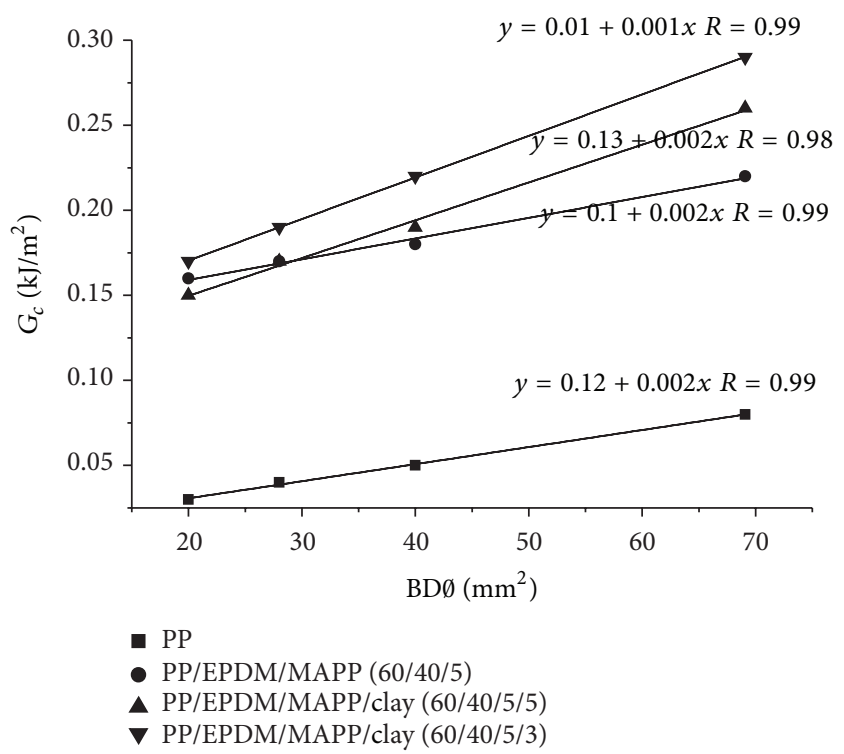

(a)

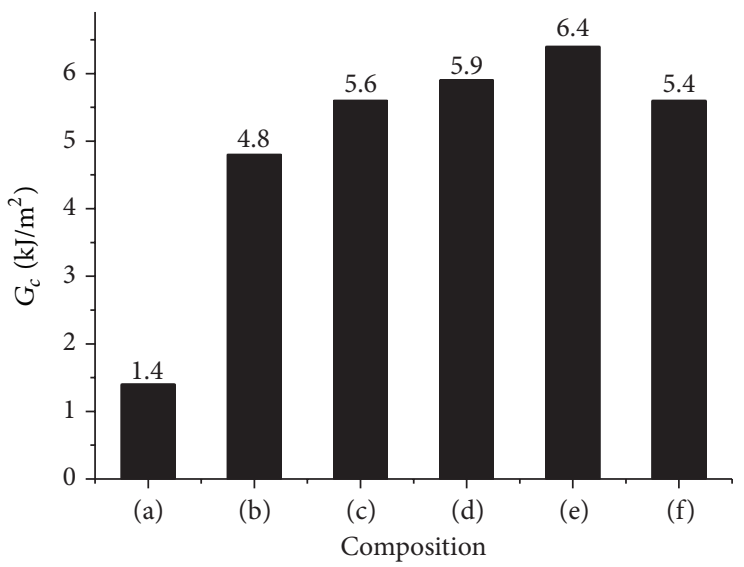

(b)

FIGURE 4: (a) Plots of total energy versus "corrected" ligament area for Izod impact test. (b) Critical energy strain release rate " $G_{c}$ ” of (a) VPP (b) PP/EPDM (60/40), (c) PP/EPDM/MAPP (60/40/5) blend, (d) PP/EPDM/MAPP/Clay (1 wt\%), (e) PP/EPDM/MAPP/Clay (3 wt\%), (f) $\mathrm{PP} / \mathrm{EPDM} / \mathrm{MAPP} / \mathrm{Clay}(5 \mathrm{wt} \%)$ blend nanocomposites.

Fracture toughness value, $G_{c}$, of PP polymer continuously increased significantly from $1.4 \mathrm{KJ} / \mathrm{m}^{2}$ for $\mathrm{PP}$ to $6.4 \mathrm{KJ} / \mathrm{m}^{2}$ and $5.6 \mathrm{KJ} / \mathrm{m}^{2}$, respectively, for PP/EPDM/MAPP nanocomposite at $3 \mathrm{wt} \%$ and $5 \mathrm{wt} \%$ clay loading. This further indicated that presence of MAPP as compatibilizer with clay induced impact toughness on the elastomer modified PP (Figure 4(b)). Maximum improvement of $G_{c}$ was 4.5 times and 4 times, respectively, which was observed in the $3 \mathrm{phr}$ and $5 \mathrm{wt} \%$ nanoclay filled PP composite when compared with the virgin PP polymer. Even though the addition of nanoclay continuously increased the $G_{c}$ values, the rate of increase was slower at higher clay concentration. The exfoliated structures contributed more toughness properties towards the loading directions due to the increase of the net aspect ratio. The increased value of $G_{c}$ suggested better stress transfer across the interfaces of blend containing rubber. However, a decrease of $G_{c}$ value has been observed with further increase of nanoclay. It might be due to the nanoclay particles that interacted with each other and formed aggregations.

Developed aggregates promote formation of premature crack due to poor adhesion between nanoparticles and matrix which reduced toughness of the nanocomposites. From the above, it can be concluded that $G_{c}$ value for nanocomposite is very large as compared to VPP which depicted that large amount of energy dissipated during fracture per unit of newly created fracture surface area which results in more fracture toughness of the blend nanocomposites. As depicted from Figure 4(a), all slopes of the nanocomposites and blends provided strong $R$ curve behavior $(R>0.9)$. Both $3 \mathrm{wt} \%$ and $5 \mathrm{wt} \%$ clay loading result in sharp slope with higher $G_{c}$ value which indicated more substantial resistance to crack growth. This may be due to the added elastomer in the nanocomposite undergoing cavitation due to crack propagation, which may release the triaxiality at the notch and permits matrix deformations by shear yielding. It is worthy to note that addition of clay as filler is expected to make high embrittlement to the elastomer modified PP matrix.

4.3.2. J-Integral Approach. Figure 5(a) indicates specimen geometry used having varying crack sizes for the measurement of $J$-integral based on the construction of the locus of crack initiation points as determined on the corresponding load-displacement records measured in a sample of SENT experiments. It has been successfully applied to characterize the $J_{c}$ value for highly deformable materials without restricting the specimen geometry. The $a / w$ (initial crack/width) ratio was varied from 0.2 to 0.3 . Load-displacement graphs were recorded and crack initiation points were marked on each loading line during the test. The crack tip area was illuminated from behind the fixture so that the crack initiation points could be clearly seen. Due to slow crosshead speed, there was ample time to respond to the crack initiation process. Similar to the crack initiation point, maximum load point in the load versus deflection curves can be used to measure fracture toughness using (2) (R-curves). Here $U_{c}$ is replaced with $U_{R}$ which is the area enclosed by the locus line of maximum points of the load-displacement curves and the $x$-axis. For brittle materials, the maximum load point coincides with the crack initiation point, whereas for ductile materials this may not be the case. For ductile materials the maximum point can be easily identified as there is a sharp transition in the crack growth resistance curves ( $R$-curves) [29]. 


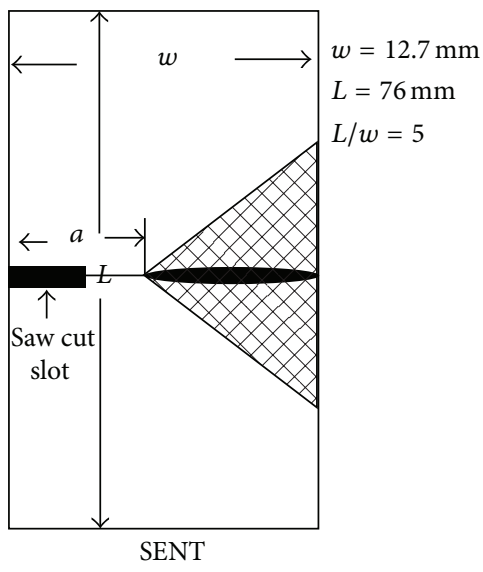

(a)

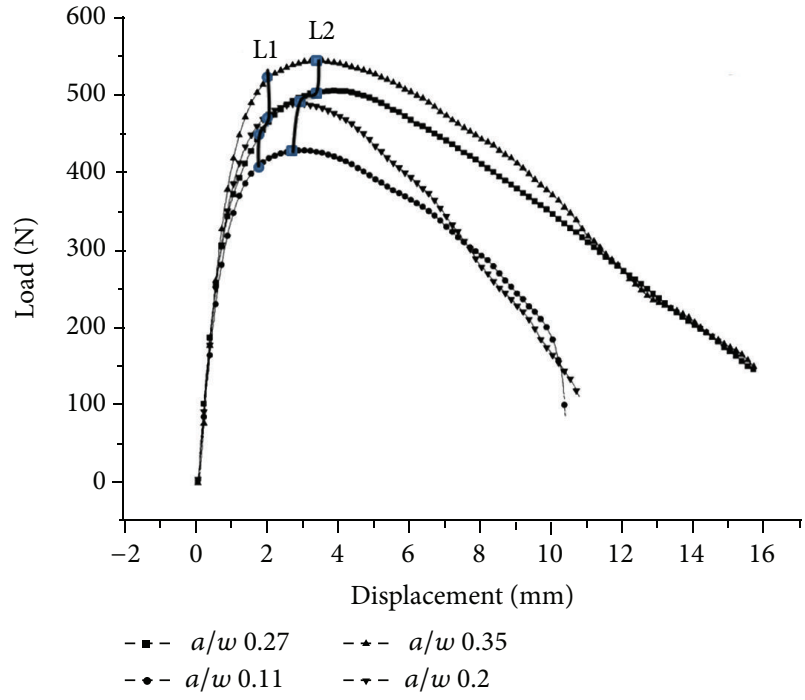

(b)

FIGURE 5: (a) Geometry of SENT specimen showing plastic yielding zone. (b) Load-displacement curves of SENT specimens with different $a / w$ ratios of $\mathrm{PP} / \mathrm{EPDM}$ blend nanocomposite with $3 \mathrm{wt} \%$ clay.

Figure 5 shows the typical load-displacement curves for PP/EPDM blend nanocomposite with $3 \mathrm{wt} \%$ clay at different crack lengths. The filled circles as denoted in the graph indicated crack initiation points and line L1 denotes the locus of the crack initiation. The shape of the locus is dependent on the specimen length since the total deformation energy in the specimens prior to crack initiation is also dependent on the specimen length. Since the locus method determines $J_{c}$ by partitioning the essential energy required for crack propagation, consistent $J_{c}$ values are obtained regardless of the specimen length. The linear least square fitted slope was taken as $J_{c}$. In Figure 5(b), rectangular circles denote the observed maximum load for crack growth and line L2 denotes the locus of maximum load points. Moreover, most specimens manifested evident necking after yielding. Since the forcedisplacement curves of specimens with different ligament lengths were geometrically similar (self-similarity), the fracture mechanism was probably independent of the ligament length. Interestingly, in all samples including nanocomposite, the area under the curve after the maximum force is higher than that prior to maximum force, thus indicating slow crack propagation with high energy absorption, typical of ductile materials [30]. The specimens exhibited ductile failure with gross yielding and necking. The presence of the plane stress deformation is apparent by the contraction of the specimen surfaces.

Figures 6 and 7 show the variation of essential energy needed for crack initiation per unit thickness $\left(U_{c} / B\right)$ and crack growth resistance at maximum load per unit thickness $\left(U_{R} / B\right)$ with initial crack length $(a)$, respectively. In the plot of $U_{R} / B$ versus crack length, all the specimens exhibited delayed yielding (i.e., the ligament yielding is fully yielded when the crack starts to propagate), with subsequent ductile fracture, showing a large plastic deformation zone surrounding crack tip. The result shows that for a given sample thickness the $J$ value at the crack initiation point $\left(J_{c}\right)$ is constant and $R$ values at the maximum crack resistant point $\left(R_{\max }\right)$ are constant. As shown in Figure $8, R_{\max }$ values are higher than the $J_{c}$ values. For these blends, the maximum load point occurs well after the observed crack initiation point. $R_{\max }$ characterises the fracture event associated with a certain amount of crack advancement at the maximum load point. This suggested ductile behavior of the nanocomposite and blend materials. $R_{\max }$ is always higher than $J_{c}$ due to inherent increase in crack resistance associated with the crack growth. Figure 8 also shows that PP/EPDM blend with $3 \mathrm{wt} \%$ clay shows the maximum $J_{c}$ and $R_{\max }$ values and, hence, better fracture toughness than the other compositions as studied.

The addition of clay along with compatibilizer increased the toughness of the nanocomposite. The effect of elastomer and clay composition on $J_{c}$ and $R_{\max }$ can be explained on the basis of interactions between the clay and elastomer particles. At low concentration of clay, lower $J_{c}$ value was observed due to insignificant interaction between the particles until very large plastic strains have been reached. As the amount of clay concentration has been increased, the clay particles will begin to interact with compatibilizer along with elastomers after a moderate amount of plastic strain. With further inclusion of clay, aggregation may result and the diameter of the ligament between the rubber particles reduces even more by hole growth, which reduces the load carrying capacity. Consequently, the critical value for crack initiation $J_{c}$ and critical value at maximum load for crack growth, $R_{\max }$, decrease by increasing the clay concentration in the blends [31].

4.4. Fracture Surface Analysis Using TEM. Figure 9 shows TEM micrographs of PP/EPDM/MAPP(60/40/5) blends 


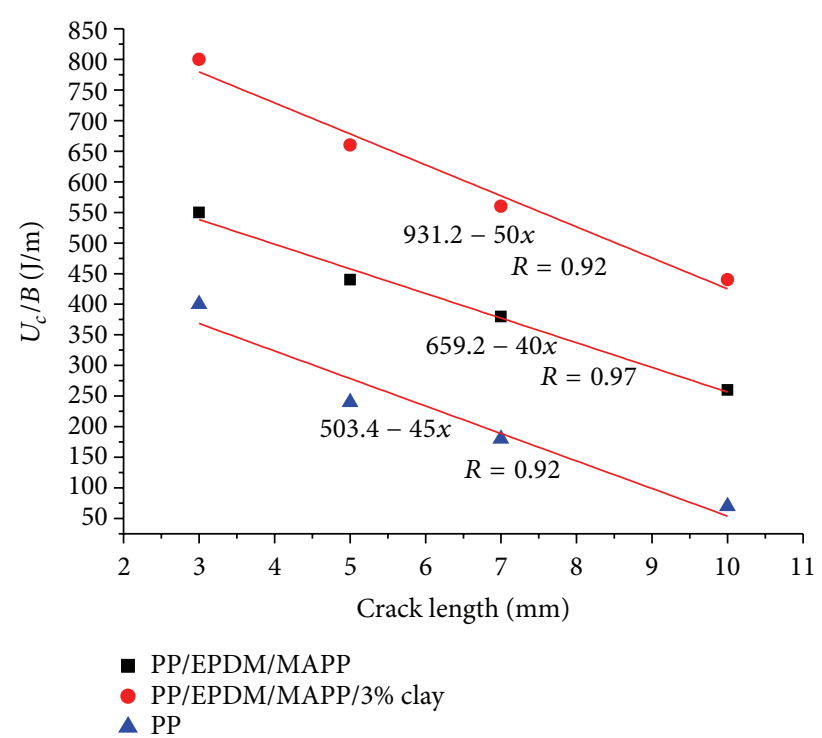

FIGURE 6: Crack initiation energy per unit thickness and $U_{c} / B$ versus crack length of PP/EPDM/MAPP blend and PP/EPDM/ $\mathrm{MAPP} / 3 \mathrm{wt} \%$ clay nanocomposite.

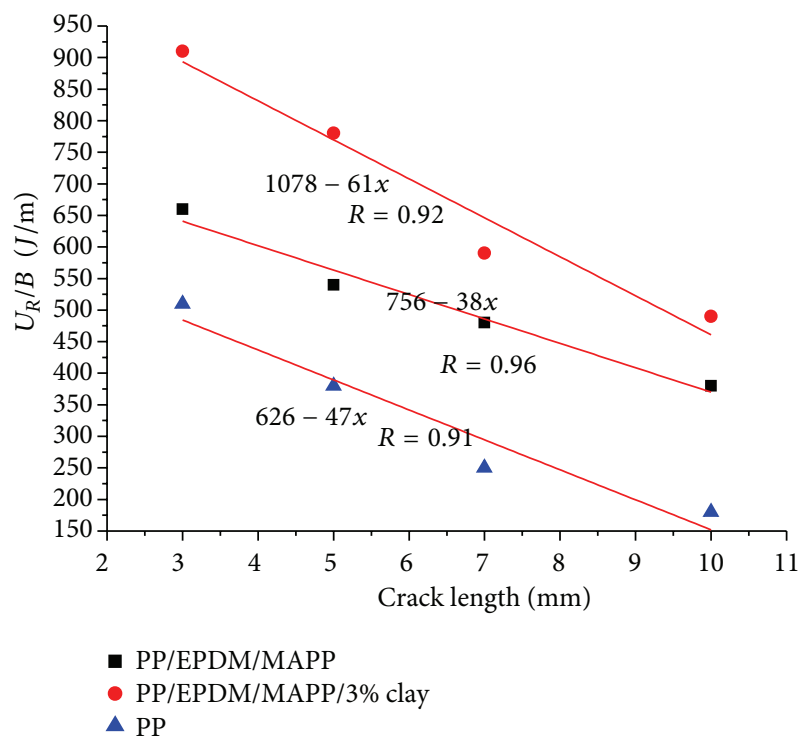

FIgURE 7: Crack growth resistance at maximum load per unit thickness and $U_{R} / B$ versus crack length of PP/EPDM/MAPP blend and $\mathrm{PP} / \mathrm{EPDM} / \mathrm{MAPP} / 3 \mathrm{wt} \%$ clay nanocomposite.

and nanocomposite containing $3 \mathrm{wt} \%$ of clay taken from the impact fractured specimen. In TEM micrographs of PP/EPDM/MAPP (60/40/5) blends with no clay, elongated elastomer particles ranging in size from 4 to $7 \mu \mathrm{m}$ were observed (Figure 9(a)). The elastomer particles that are still much larger can be easily identified and appear quite homogeneously dispersed in the PP matrix. On the other hand, nanocomposite containing $3 \mathrm{wt} \%$ clay as observed from Figure 9(b) reveals a mixed morphological structure, that is, combination of intercalated stacks and exfoliated particles on

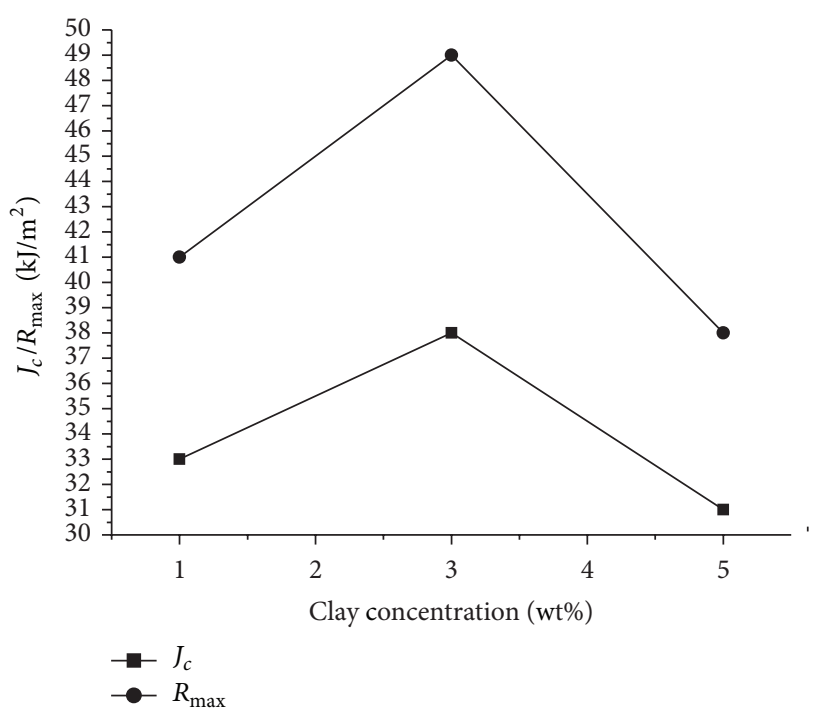

FIGURE 8: $J_{c} / R_{\max }$ value of the blend nanocomposites as a function of clay content.

the surface. Also, it is noted that a large portion of the clay particles appear as bent, skewed, and/or curved structures when viewed parallel to transverse direction. As evident from the TEM micrographs, the small elastomeric particles are uniformly distributed in the PP matrix. In addition, the micrographs showed more homogeneous morphology with fine and uniform dispersion of elastomer particles in the continuous PP matrix. Moreover, more reduced size of the dispersed rubber particles was observed by addition of $3 \mathrm{wt} \%$ clay and MAPP grafted polymers. These results suggest that grafted MAPP compatibilizer with presence of clay has a significant effect on the rubber particle size and possibly on the interfacial adhesion. This is one of the aspects responsible for the better impact behavior of nanocomposites as evident from the toughness results. A decrease of the rubber particles size is correlated with the interfacial adhesion between both polymeric phases; thus the interfacial tension decreases. Mirabella et al. also reported that addition of organoclay caused a reduction of rubber particle size in $\mathrm{PP} / \mathrm{elastomer}$ blends [32]. They attributed the apparent reduction in rubber particle size to the increase in melt viscosity as clay loading increases and/or the role of clay as a chemical modifier for the rubber phase. A similar reduction in elastomer particle size resulted in the developed nanocomposite system may be due to higher forces induced by the higher melt viscosity as clay content increases.

A conceptual illustration of this proposed theory and subsequent failure mechanism proposed that the clay nanoplatelets are acting as very redirected stress concentrations away from the elastomeric impact modification (toughening) phase. This will not allow the conventional toughening mechanisms to occur. Also it is expected that stress concentrations instead occur around the clay because of the much higher mismatch of modulus. The proposed failure mechanism is detailed in the following steps: (1) stress concentrations occur surrounding the clay platelets, (2) rapid 


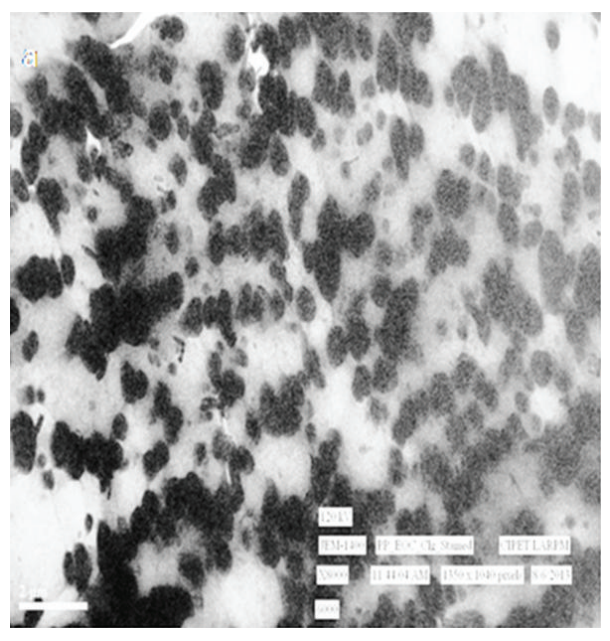

(a)

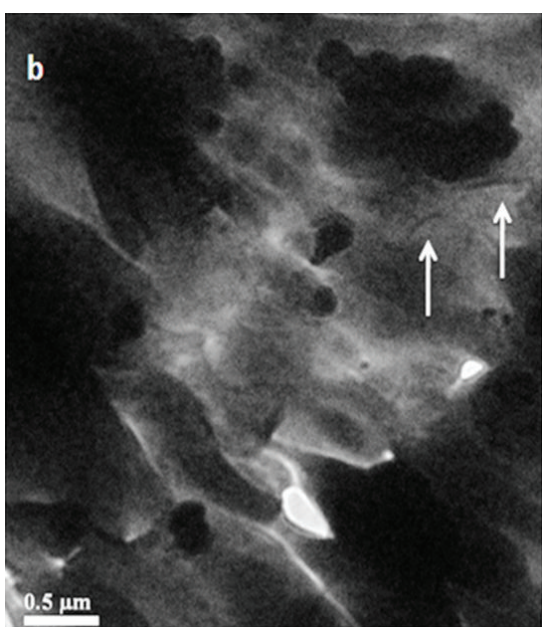

(b)

FIGURE 9: TEM micrograph of impact fracture surface of (a) PP/EPDM/MAPP (60/40/5) blend and (b) PP/EPDM/MAPP/Clay (60/40/5/3) blend nanocomposites at room temperature.

delamination of the matrix from the filler ensues because of the lack of affinity (poor adhesion) between PP and clay, and (3) catastrophic crack propagation can occur with little energy applied.

\section{Conclusion}

PP polymer filled with EPDM elastomer and nanoclay at various concentrations ( 1,3 , and $5 \mathrm{phr}$ ) was prepared by using melt blending extrusion method. MAPP has been used as the compatibilizer to promote interfacial adhesion between the clay and the matrix.

(i) The presence of $40 \%$ elastomer reasonably increased impact strength up to $175 \%$ compared to neat PP which resulted in phenomenal transition from brittle to ductile behavior of PP. Impact strength of blend matrix increased up to $660 \mathrm{MPa}$ which indicates toughening effect of nanoclay layers within the blend matrix.

(ii) The value of fracture energy, $G_{c}$, for all blends and nanocomposites generally increases as the crack propagated; that is, a rising of " $R$-curve" is observed. The increase of $G_{c}$ reveals that there is significant improvement in toughness.

(iii) Fracture toughness studied using the $J$-integral by locus method revealed maximum toughness value at $3 \mathrm{wt} \%$ clay loading.

(iv) TEM micrograph reveals formation of both intercalated and exfoliated structure with significant changes in blend morphology and toughness which were obtained with reduction in dispersed particle size and an increase in adhesion between the phases formation.

\section{Conflict of Interests}

The authors do not have any conflict of interests regarding the company/material names mentioned in the paper. There is no financial interest in the outcome of the results or failure of any of the used materials in the paper.

\section{References}

[1] N. Inaba, K. Sato, S. Suzuki, and T. Hashimoto, "Morphology control of binary polymer mixtures by spinodal decomposition and crystallization. 1: principle of method and preliminary results on PP/EPR," Macromolecules, vol. 19, no. 6, pp. 16901695, 1986.

[2] W. Jiang, S. C. Tjong, and R. K. Y. Li, "Brittle-tough transition in PP/EPDM blends: effects of interparticle distance and tensile deformation speed," Polymer, vol. 41, no. 9, pp. 3479-3482, 2000.

[3] R. R. Babu, N. K. Singha, and K. Naskar, "Interrelationships of morphology, thermal and mechanical properties in uncrosslinked and dynamically crosslinked PP/EOC and PP/EPDM blends," Express Polymer Letters, vol. 4, no. 4, pp. 197209, 2010.

[4] T. McNally, P. McShane, G. M. Nally, W. R. Murphy, M. Cook, and A. Miller, "Rheology, phase morphology, mechanical, impact and thermal properties of polypropylene/metallocene catalysed ethylene 1-octene copolymer blends," Polymer, vol. 43, no. 13, pp. 3785-3793, 2002.

[5] L. Zhang, C. Li, and R. Huang, “Toughness mechanism in polypropylene composites: polypropylene toughened with elastomer and calcium carbonate," Journal of Polymer Science B, vol. 42, no. 9, pp. 1656-1662, 2004.

[6] G. P. Marshall, J. G. Williams, and C. E. Turner, "Fracture toughness and absorbed energy measurements in impact tests on brittle materials," Journal of Materials Science, vol. 8, no. 7, pp. 949-956, 1973.

[7] E. Plati and J. G. Williams, "The determination of the fracture parameters for polymers in impact," Polymer Engineering and Science, vol. 15, no. 6, pp. 470-477, 1975. 
[8] C. G. Sanporean, Z. Vuluga, J. D. Christiansen, C. Radovici, E. A. Jensen, and H. Paven, "Investigation of mechanical properties of PP/Clay nanocomposites based on network crosslinked compatibilizers," Industrial and Engineering Chemistry Research, vol. 52, no. 10, pp. 3773-3778, 2013.

[9] J. R. Rice and D. M. Tracey, "On the ductile enlargement of voids in triaxial stress fields," Journal of the Mechanics and Physics of Solids, vol. 17, no. 3, pp. 201-217, 1969.

[10] B. H. Kim and C. R. Joe, "A method to evaluate critical J-integral value: locus method," Polymer Testing, vol. 7, no. 5, pp. 355-363, 1987.

[11] B. H. Kim, C. R. Joe, and D. M. Otterson, "On the determination of fracture toughness in polymers," Polymer Testing, vol. 8, no. 2, pp. 119-130, 1988.

[12] Y. S. Lee, W.-K. Lee, S.-G. Cho, I. Kim, and C.-S. Ha, "Quantitative analysis of unknown compositions in ternary polymer blends: a model study on NR/SBR/BR system," Journal of Analytical and Applied Pyrolysis, vol. 78, no. 1, pp. 85-94, 2007.

[13] P. Sivaraman, L. Chandrasekhar, V. S. Mishra, B. C. Chakraborty, and T. O. Varghese, "Fracture toughness of thermoplastic co-poly (ether ester) elastomer-Acrylonitrile butadiene styrene terpolymer blends," Polymer Testing, vol. 25, no. 4, pp. 562-567, 2006.

[14] A. Hassan, M. U. Wahit, and C. Y. Chee, "Mechanical and morphological properties of PP/NR/LLDPE ternary blend: effect of HVA-2," Polymer Testing, vol. 22, no. 3, pp. 281-290, 2003.

[15] H. Anuar, S. B. Abd Razak, N. M. Kahar, and N. A. Jamal, "Effects of high energy radiation on mechanical properties of PP/EPDM nanocomposite," Advanced Materials Research, vol. 264, pp. 738-742, 2011.

[16] L. Valentini, J. Biagiotti, J. M. Kenny, and M. A. López Manchado, "Physical and mechanical behavior of single-walled carbon nanotube/polypropylene/ethylene-propylene-diene rubber nanocomposites," Journal of Applied Polymer Science, vol. 89, no. 10, pp. 2657-2663, 2003.

[17] H.-S. Lee, P. D. Fasulo, W. R. Rodgers, and D. R. Paul, “TPO based nanocomposites. Part 1: morphology and mechanical properties," Polymer, vol. 46, no. 25, pp. 11673-11689, 2005.

[18] G. M. Shashidhara and S. K. Devi, "Studies on PP/SBS blends with and without nanoclay," Indian Journal of Engineering and Materials Sciences, vol. 18, no. 1, pp. 69-78, 2011.

[19] G. Jannerfeldt, L. Boogh, and J.-A. E. Månson, “Tailored interfacial properties for immiscible polymers by hyperbranched polymers," Polymer, vol. 41, no. 21, pp. 7627-7634, 2000.

[20] R. W. Sandoval, D. E. Williams, J. Kim, C. B. Roth, and J. M. Torkelson, "Critical micelle concentrations of block and gradient copolymers in homopolymer: effects of sequence distribution, composition, and molecular weight," Journal of Polymer Science B, vol. 46, no. 24, pp. 2672-2682, 2008.

[21] R. Asaletha, P. Bindu, I. Aravind et al., "Stress-relaxation behavior of natural rubber/polystyrene and natural rubber/polystyrene/natural Rubber-graft-polystyrene blends," Journal of Applied Polymer Science, vol. 108, no. 2, pp. 904-913, 2008.

[22] W. S. Chow, Z. A. M. Ishak, J. Karger-Kocsis, A. A. Apostolov, and U. S. Ishiaku, "Compatibilizing effect of maleated polypropylene on the mechanical properties and morphology of injection molded polyamide 6/polypropylene/organoclay nanocomposites," Polymer, vol. 44, no. 24, pp. 7427-7440, 2003.

[23] D. M. Bigg, "Mechanical properties of particulate filled polymers," Polymer Composites, vol. 8, no. 2, pp. 115-122, 1987.
[24] T. B. Lewis and L. E. Nielsen, "Dynamic mechanical properties of particulate-filled composites," Journal of Applied Polymer Science, vol. 14, no. 6, pp. 1449-1471, 1970.

[25] G. Erhard, Designing with Plastics, Hanser, Munich, Germany, 2006.

[26] E. Vassileva and K. Friedrich, "Epoxy/alumina nanoparticle composites. I: dynamic mechanical behavior," Journal of Applied Polymer Science, vol. 89, no. 14, pp. 3774-3785, 2003.

[27] K. T. Gam, M. Miyamoto, R. Nishimura, and H.-J. Sue, "Fracture behavior of core-shell rubber-modified clay-epoxy nanocomposites," Polymer Engineering and Science, vol. 43, no. 10, pp. 1635-1645, 2003.

[28] K. L. Johnson and J. A. Greenwood, "An adhesion map for the contact of elastic spheres," Journal of Colloid and Interface Science, vol. 192, no. 2, pp. 326-333, 1997.

[29] C.-S. Ha, Y. Kim, and W.-J. Cho, "Fracture toughness investigation of the dynamically vulcanized EPDM/PP/ionomer ternary blends using the $J$-integral via the locus method," Journal of Materials Science, vol. 31, no. 11, pp. 2917-2924, 1996.

[30] H. G. Tattersall and G. Tappin, "The work of fracture and its measurement in metals, ceramics and other materials," Journal of Materials Science, vol. 1, no. 3, pp. 296-301, 1966.

[31] D. S. Parker, H.-J. Sue, J. Huang, and A. F. Yee, “Toughening mechanisms in core-shell rubber modified polycarbonate," Polymer, vol. 31, no. 12, pp. 2267-2277, 1990.

[32] F. M. Mirabella Jr., S. P. Westphal, P. L. Fernando, E. A. Ford, and J. G. Williams, "Morphological explanation of the extraordinary fracture toughness of linear low density polyethylenes," Journal of Polymer Science B, vol. 26, no. 9, pp. 1995-2005, 1988. 

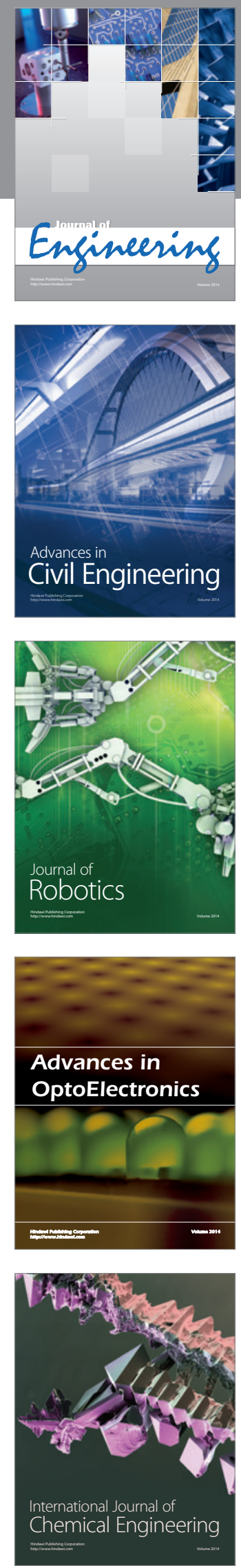

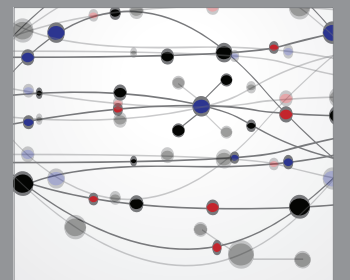

The Scientific World Journal
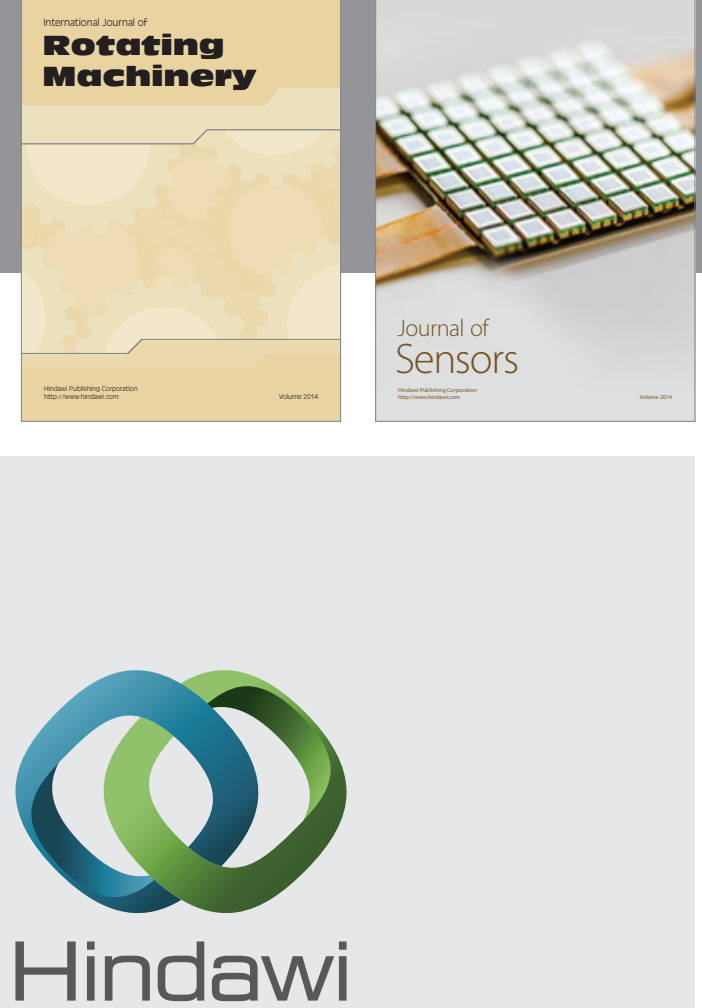

Submit your manuscripts at http://www.hindawi.com
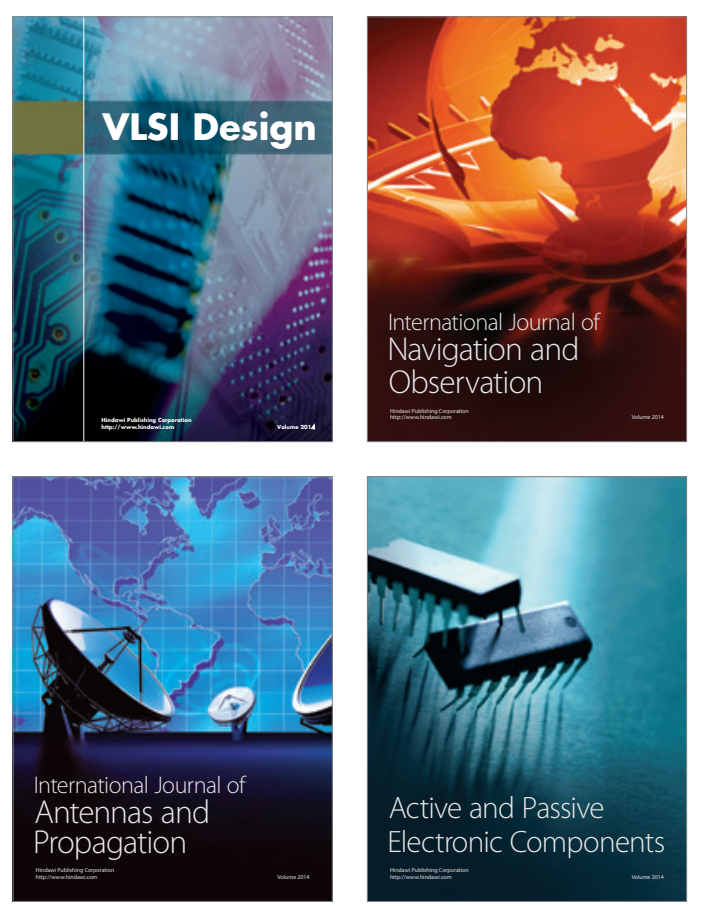
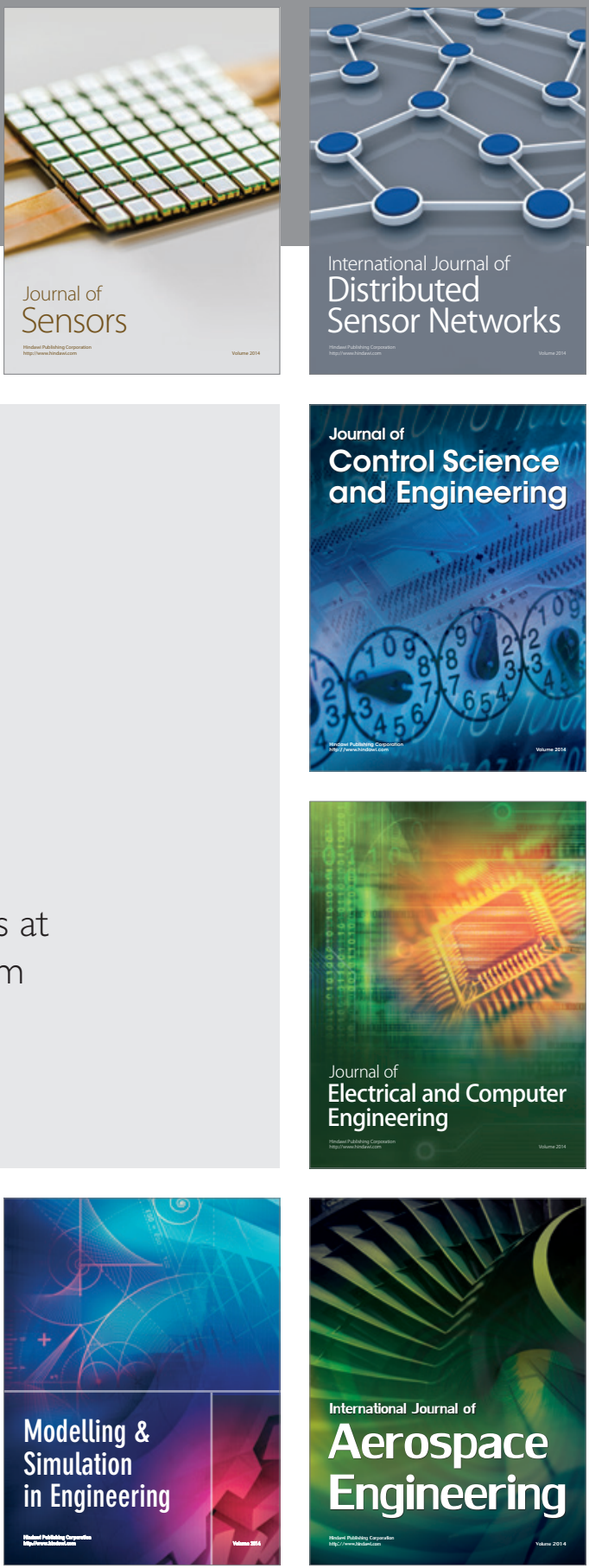

Journal of

Control Science

and Engineering
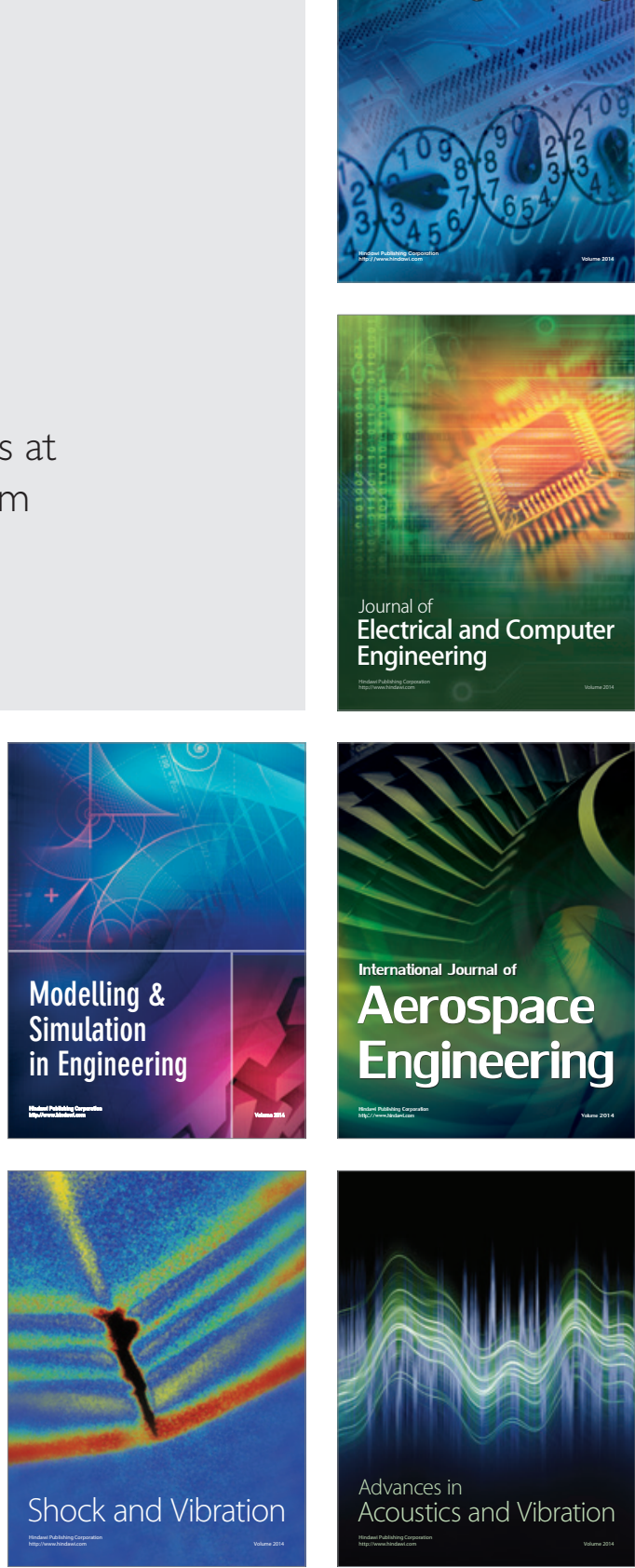